\title{
An Image-Based Impact Test for the High Strain Rate Tensile Properties of Brittle Materials
}

\author{
Lloyd Fletcher $^{1, *}$ and Fabrice Pierron ${ }^{1, * *}$ \\ ${ }^{1}$ Engineering and the Environment, University of Southampton, Highfield campus, University Road, Southampton SO17 1BJ, UK
}

\begin{abstract}
Testing ceramics at high strain rates presents many experimental difficulties due to the brittle nature of the material being tested. When using a split Hopkinson pressure bar (SHPB) for high strain rate testing, adequate time is required for stress wave effects to dampen out. For brittle materials, with small strains to failure, it is difficult to satisfy this constraint. Because of this limitation, there are minimal data (if any) available on the stiffness and tensile strength of ceramics at high strain rates. Recently, a new image-based inertial impact (IBII) test method has shown promise for analysing the high strain rate behaviour of brittle materials. This test method uses a reflected compressive stress wave to generate tensile stress and failure in an impacted specimen. Throughout the propagation of the stress wave, full-field displacement measurements are taken, from which strain and acceleration fields are derived. The acceleration fields are then used to reconstruct stress information and identify the material properties. The aim of this study is to apply the IBII test methodology to analyse the stiffness and strength of ceramics at high strain rates. The results show that it is possible to identify the elastic modulus and tensile strength of tungsten carbide at strain rates on the order of $1000 \mathrm{~s}^{-1}$. For a tungsten carbide with $13 \%$ cobalt binder the elastic modulus was identified as $516 \mathrm{GPa}$ and the strength was $1400 \mathrm{MPa}$. Future applications concern boron carbide and sapphire, for which limited data exist in high rate tension.
\end{abstract}

\section{Introduction}

Testing ceramics at high strain rates presents many experimental difficulties due to the brittle nature of the material being tested. When using a split Hopkinson pressure bar (SHPB) for high strain rate testing, adequate time is required for stress wave effects to dampen out. For brittle materials, with small strains to failure, it is difficult to satisfy this constraint. Thus, available high strain rate data for ceramics mainly concern compression [1], even though compression failure is always tensile (generally through shear) or structural (buckling). In fact, in [1], compression is applied to a dumbbell specimen but failure is initiated in the transition zone of the dumbbell shape, most likely due to shear. Therefore, there is a strong need for well-founded tensile fracture data at high strain rates for ceramics, so that structural compression failure can be predicted from the tensile strength.

The idea of using wave reflection to produce tension is not new. So-called 'spalling tests' have been around for a long time, though this terminology encompasses very different realities, from shock-based plate impact spalling on metal [2] to lower rate spalling of concrete [3] or composites $[4,5]$. Classical data interpretation of the spalling test relies on measurement of the velocity of the free back face of the test specimen. However, new full-field data processing techniques have recently emerged as an alternative. In [6], the Virtual Fields Method (VFM) was used for the first

\footnotetext{
*e-mail: L.C.Fletcher@ soton.ac.uk

**e-mail: f.pierron@soton.ac.uk
}

time on concrete spall tests using the configuration from [3]. The acceleration field obtained by temporal differentiation was used to reconstruct transverse stress averages, thus producing stress-strain curves in transverse sections without the need for any external force measurement.

The tests in $[6,7]$ are uni-axial in nature (cylindrical test specimen) but the idea can easily be extended to planar specimens [8]. Recently, both intra [9, 10] and interlaminar [9] tensile properties of composites were successfully obtained with this new methodology, named Image-Based Inertial Impact (IBII). The objective of the present paper is to show how this methodology can be extended to very strong materials like ceramics and cermets. This is illustrated here on Tungsten carbide with a cobalt-binder.

\section{Experimental set-up and data processing}

\subsection{Experiment}

The material under study here is fine grain tungsten carbide with a cobalt binder (General Carbide GC-313 grade). The binder has a volume fraction of approximately $12.5 \%$ and the average grain size is around $1 \mu \mathrm{m}$. The experiment consists in inertially impacting a thin rectangular test specimen, as shown in figure 1 . The specimen was bonded onto a cylindrical waveguide made of high strength steel (alloy 15CDV6). This ensured good contact with the specimen and helped to input a clean stress wave. The waveguide was impacted by short projectile made from the same high 
strength steel and mounted into an oil filled nylon (Oilon) sabot (figure 1). The projectile/sabot assembly was accelerated in a compressed air gas gun as seen in figure 2 . The impact speed, measured by a set of two light gates at the end of the barrel, was $40 \mathrm{~m} . \mathrm{s}^{-1}$. During the test, the specimen was first subjected to a compressive stress wave. When the wave reached the free edge, it reflected as a tensile wave, as in the classical spall configuration. Contrary to [6] where a long pulse was used to obtain a nearly uniform stress state where the specimen fails, the short impactor used here produced a narrow spatial stress wave which enabled higher stresses to be reached to fracture this very strong material.

As in [6], the specimen was mounted with a bidirectional grid using the transfer procedure detailed in [11], with a pitch of $0.7 \mathrm{~mm}$. The pitch was selected to obtain a sampling of 5 pixels per period since the camera has a spatial resolution of only $400 \times 250 \mathrm{pixel}^{2}$. Some free pixels were kept in the $\mathrm{x}$-direction to account for some rigid body translation during the test as it is essential to keep the free edge within the field of view. Lighting was provided by a Bowens 1000 flash light. Since the flash rise time is $100 \mu \mathrm{s}$, it was triggered off the light gates and the appropriate time delay was directly calculated using an Arduino-based custom-made system. The specimen deformation was filmed using a Shimadzu HPV-X camera at 5 Mfps. The 128 frames recorded during the test covered a record time of $25.6 \mu \mathrm{s}$, which was slightly larger than the time needed for one wave return (wavespeed of about $\left.6 \mathrm{~km} . \mathrm{s}^{-1}\right)$. Because of the short record time, the camera could not be triggered off the light gates as there would be too much uncertainty in the projectile to wageguide contact time. Therefore, two thin copper films were mounted to the wageguide, as seen in figures 1 and 2, and the circuit closure caused by the projectile impact was used to provide the trigger pulse to the camera. A delay was used to account for wave propagation in the waveguide. This ensured stable and reproducible triggering of the camera.

\subsection{Data processing}

The grid images were processed using the spatial phase shifting algorithm described in [12]. In particular, the iterative phase calculation was selected, which helps to account for slight grid defects. Instead of subtracting the reference and deformed phase maps directly, the deformed one is modified to take into account the displacement of the related material point. This was helpful to measure the small strain levels recorded here. The two in-plane components of displacement were then derived from the phase difference maps, and both spatial and temporal differentiation was implemented to calculate strains and acceleration. Because of the presence of noise in the images (and therefore, in the displacement maps), both spatial and temporal smoothing of the displacements was employed. Spatial smoothing was first applied to the displacements in the form of a local Gaussian filter over a kernel of 17 x 17 data points (about $3 \times 3$ grid lines). Strains were then obtained by centred finite differences. Temporal smoothing in the form of a third order Savitsky-Golay filter over a window of 21 data points was first applied to the raw displacements before spatial smoothing and temporal differentiation by centred finite differences to obtain acceleration. These parameters were found to provide adequate compromise between random and systematic errors. However, a systematic study based on the image deformation procedure reported in [13] is currently under way to determine optimal smoothing parameters and estimate identification uncertainties.

Using the same procedure as in [8], the average of the longitudinal stress $\sigma_{x x}$ across the specimen width, ${\overline{\sigma_{x x}}}^{y}$ can be obtained from the average of the $a_{x}$ acceleration over the surface between the considered section of coordinate $x$ and the free edge, ${\overline{a_{x}}}^{S}$, as:

$$
{\overline{\sigma_{x x}}}^{y}=\rho x{\overline{a_{x}}}^{S}
$$

where $\rho$ is the material density. If the material behaves elastically, it is then possible to reconstruct stress-strain curves at each section where a data point is available, in the form of averages across the width. Since spatial Gaussian smoothing resulted in biased data over one half of the kernel at the free edge, padding of the acceleration was necessary there to obtain good stress reconstruction using Eq. 1 at the stage of wave reflection. This was done using linear extrapolation.

\section{Results}

The stress-strain curves reconstructed using Eq. 1 and the average strains across the width are shown in figure 3 for eight sections between the free edge and the impact end (0 $\mathrm{mm}$ being the free edge). Poisson's ratio was identified using the Virtual Fields Method using the virtual fields from Eq. 15 in [14]. The identified Poisson's ratio was 0.23 .

The responses are remarkably linear elastic and consistent between the different sections. This is exceptional considering the very low levels of strains involved and the very high strain rates, up to $1000 \mathrm{~s}^{-1}$. Young's modulus was identified from the average of the slope of these curves over the $60 \%$ of sections at the specimen centre, to mitigate the effects of edges (low strains towards the free edge, effect of spatial smoothing). The average modulus identified over this set of sections was $516 \mathrm{GPa}$. The data are represented in figure 4 . Over the 251 data points in this figure, the stability of the identified stiffness is quite remarkable. Both $\mathrm{E}$ and $v$ obtained here compare well with quasi-static data published in [15]: $\mathrm{E}=540 \mathrm{GPa}$ and $v=0.22$ for Kennametal KF312 (12\% Co binder, density of $14,300 \mathrm{~kg} . \mathrm{m}^{-3}$, grain size less than $\left.1 \mu \mathrm{m}\right)$, as no strain rate effect on the stiffness was expected.

The strength was also identified using Eq. 1. In fact, a more elaborate version of this equation can be established using the three possible solid rigid body virtual fields. This provides a linear estimation of the stress over the width from only acceleration data, denoted SG,L [10]. The stress from SG,L can be compared to the stress obtained from 




Fig. 1. Close up view of the specimen attached to waveguide showing the camera contact trigger (left). High strength steel waveguide and projectile assembly (right).
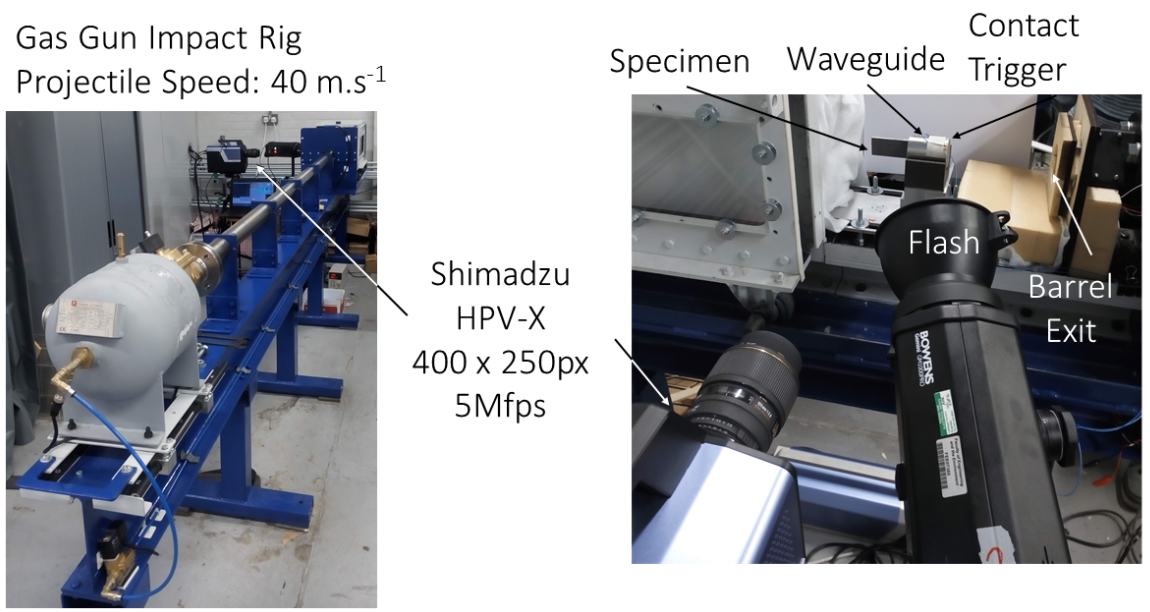

Fig. 2. Test set-up.
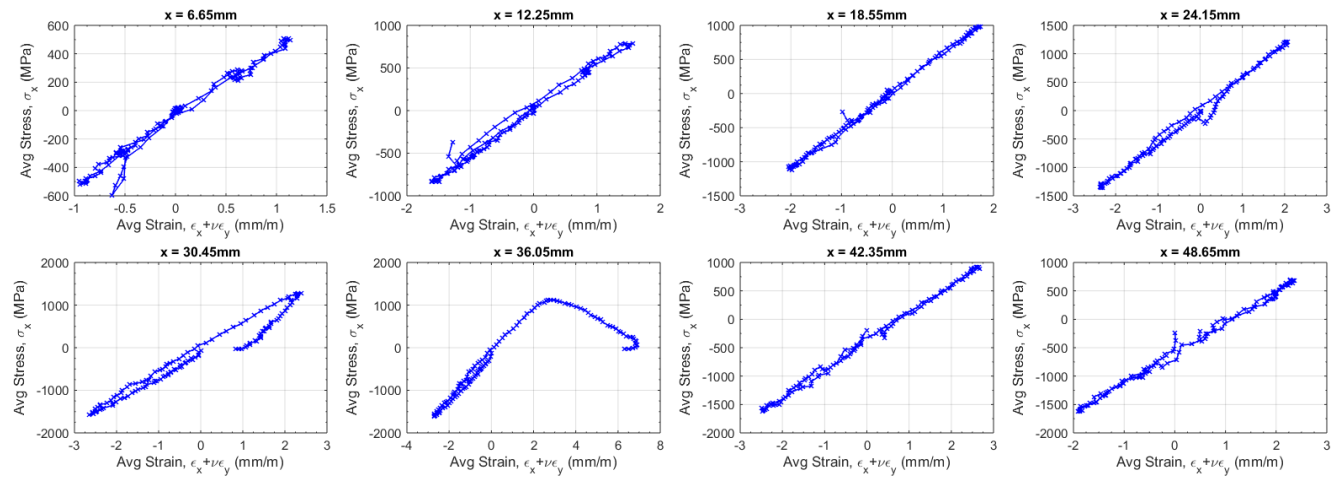

Fig. 3. Stress-strain curves at different cross-sections along the specimen length.

the strain and the identified stiffness components. Fracture will cause these two metrics to diverge. Figure 5 shows acceleration, strain and strain rate maps at the identified time step of fracture. The top-left plot shows clearly the departure of the strain-based stress from the acceleration-based stress SG,L. This enabled the identification of a tensile fracture stress of approximately $1.41 \mathrm{GPa}$. The present authors failed to find in the literature any relevant quasi-static tensile strengths on similar material systems, let alone at high rates, as all the publications reported compression or bending strengths. So one cannot comment here on the strain rate dependence of the tensile strength.

At the end of the test, the remains of the specimen were gathered and assembled. This is shown in figure 6, together with the last displacement image available at the end of the record time. One can see multi-cracking, some cracks matching the displacement map. It is not clear whether the other cracks were a consequence of the re- 


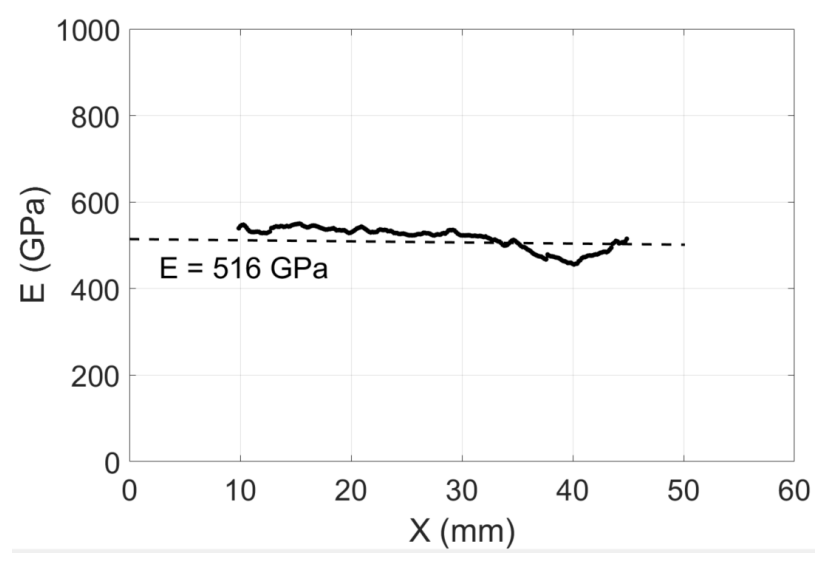

Fig. 4. Young's modulus as a function of specimen length (251 stiffness data points)

flections of the stress waves or a result of the specimen smashing into the back of the capture chamber. This could be elucidated in the future by using a lower frame rate to enable longer imaging time.

\section{Conclusion and future work}

The new IBII test protocol developed in the PhotoDyn project (www.photodyn.org) has been proved to successfully identify the stiffness and tensile strength of a tungsten carbide material at strain rates up to $1000 \mathrm{~s}^{-1}$. To the best knowledge of the authors, such high strain rate tensile results are unprecedented in the literature and provide a new and effective way to study such materials. Future work will address comparative testing of several grades of tungsten carbide, as well as extending the methodology to materials like boron carbide, which has a much higher wave speed. Uncertainty quantification and optimal smoothing parameters will be determined using synthetic grid deformation, as in [13].

The authors want to thank Dr Leslie Lamberson from Drexel University for providing the samples and for useful discussions about the material. Funding from EPSRC, grant EP/L026910/1, is also gratefully acknowledged.

\section{References}

1. J.J. Swab, C.S. Meredith, D.T. Casem, W.R. Gamble, Journal of Materials Science 52, 10073 (2017)

2. S. Zhuang, J. Lu, G. Ravichandran, Applied Physics Letters 80, 4522 (2002)

3. B. Erzar, P. Forquin, Experimental Mechanics 50, 941 (2010)

4. F. Gálvez Diaz-Rubio, J. Rodriguez Pérez, V. Sánchez Gálvez, International Journal of Impact Engineering 27, 161 (2002)

5. R.A. Govender, L.A. Louca, A. Pullen, A.S. Fallah, G.N. Nurick, Journal of Composite Materials 46, 1219 (2012)

6. F. Pierron, P. Forquin, Strain 28, 388 (2012)

7. P. Forquin, B. Lukić, Journal of Dynamic Behavior of Materials (2017)

8. F. Pierron, H. Zhu, C. Siviour, Phil. Trans. A 372, 20130195 (2014)

9. L. Fletcher, F. Pierron, Inertial impact method for the through-thickness strength of composites, in Conference Proceedings of the Society for Experimental Mechanics Series, edited by Springer (????), pp. 105-108

10. L. Fletcher, J. Van Blitterswyk, F. Pierron, Composites Part A: Applied Science and Manufacturing Submitted. (2018)

11. J.L. Piro, M. Grediac, Experimental Techniques 28, 23 (2004)

12. M. Grédiac, F. Sur, B. Blaysat, Strain 52, 205 (2016)

13. M. Rossi, P. Lava, F. Pierron, D. Debruyne, M. Sasso, Strain 51, 206 (2015)

14. R. Moulart, F. Pierron, S.R. Hallett, M.R. Wisnom, Experimental Mechanics 51, 509 (2011)

15. I.C. Getting, G. Chen, J.A. Brown, pure and applied geophysics 141, 545 (1993) 


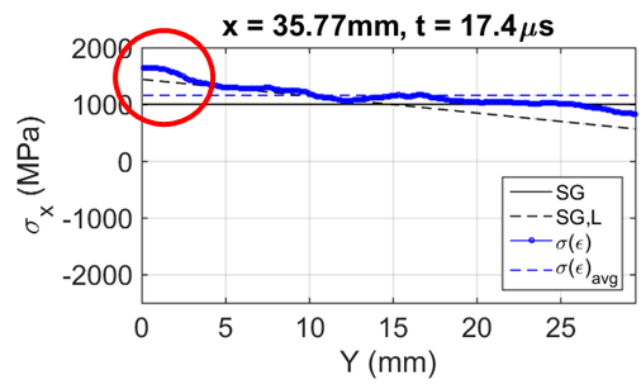

$\epsilon_{x}\left[\mathrm{~mm} \cdot \mathrm{m}^{-1}\right]$

$\mathrm{t}=17.40 \mu \mathrm{s}$

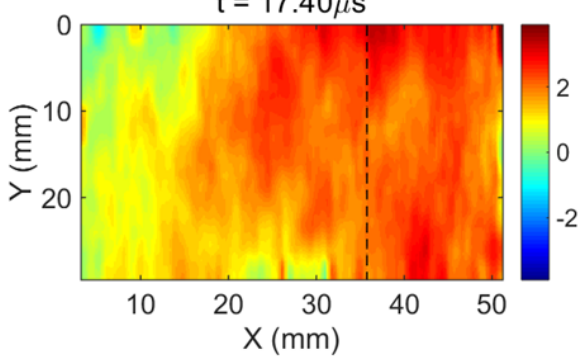

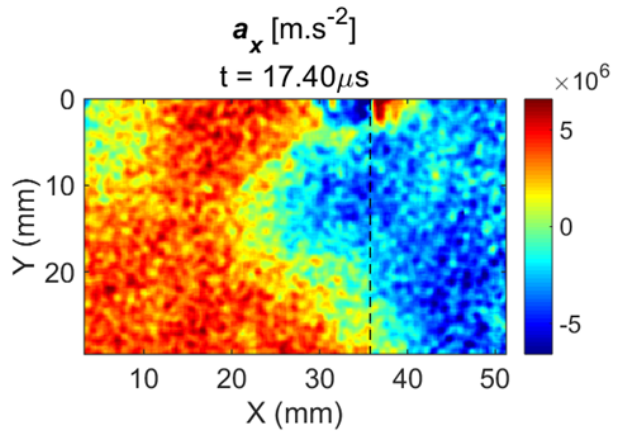

$d \epsilon_{x} / d t\left[\mathrm{~s}^{-1}\right]$

$\mathrm{t}=17.40 \mu \mathrm{s}$



Fig. 5. Fracture diagnostics maps: strain, strain rate, acceleration and stress-gauge curves

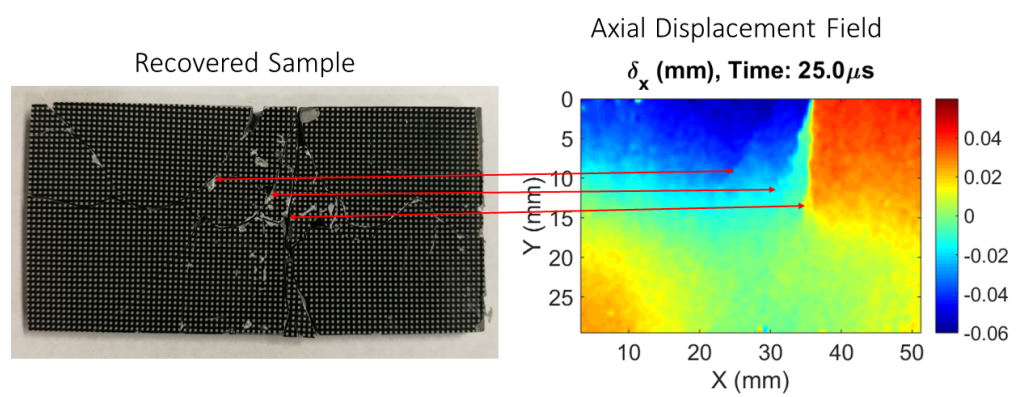

Fig. 6. Fractured specimen with axial displacement at the last time step. 
